\title{
The Dose-Related Effects of Extracorporeal Shock Wave Therapy for Knee Osteoarthritis
}

\author{
Jin-Hong Kim, MD, Ja-Young Kim, MD, Cheol-Min Choi, MD, June-Kyung Lee, MD, \\ Hoi-Sung Kee, MD, Kwang-Ik Jung, MD, Seo-Ra Yoon, MD
}

Department of Rehabilitation Medicine, Gwangju Veterans Hospital, Gwangju, Korea

\begin{abstract}
Objective To investigate the dose-related effects of extracorporeal shock wave therapy (ESWT) for knee osteoarthritis.

Methods Seventy-five subjects were recruited, 60 of which met the inclusion criteria. The patients were randomly classified into two groups: group $\mathrm{L}$, which was a low-energy group ( $\mathrm{n}=30 ; 1,000$ shocks/session; energy flux density [EFD], $0.040 \mathrm{~mJ} / \mathrm{mm}^{2}$ ) and group $\mathrm{M}$, which was a medium-energy group ( $\mathrm{n}=30 ; 1,000$ shocks/session; EFD, 0.093 $\mathrm{mJ} / \mathrm{mm}^{2}$ ). For each group, 1,000 shock waves were delivered to the medial tibial plateau area, once a week, for 3 weeks. The main outcome measures were the visual analogue scale (VAS), the Roles and Maudsley (RM) score, the Western Ontario and McMaster Universities Osteoarthritis Index (WOMAC) score, and the Lequesne index. Each assessment was performed at the baseline and at 1, 4, and 12 weeks after ESWT.

Results In both groups, the VAS, the RM and WOMAC scores, and the Lequesne index were significantly improved over time $(\mathrm{p}<0.001)$, and group $M$ showed greater improvement over group $\mathrm{L}$ at the 1,4 and 12 weeks assessments. Conclusion In this study, medium-energy group (group M) showed greater improvement in regard to relieving pain and restoring functional outcome than the low-energy group (group L). Therefore, EFD can be considered to have significant influence when treating with ESWT for knee osteoarthritis.
\end{abstract}

Keywords Osteoarthritis, Knee, High-energy shock waves, Treatment outcome

\section{INTRODUCTION}

Osteoarthritis (OA) is the most common form of degenerative arthritis; it is a widespread, slowly developing dis-

Received February 9, 2015; Accepted April 10, 2015

Corresponding author: Seo-Ra Yoon

Department of Rehabilitation Medicine, Gwangju Veterans Hospital, 99 Cheomdanwolbong-ro, Gwangsan-gu, Gwangju 506-705, Korea

Tel: +82-62-602-6295, Fax: +82-62-602-6990, E-mail: standupmd@hanmail. net

(c) This is an open-access article distributed under the terms of the Creative Commons Attribution Non-Commercial License (http://creativecommons. org/licenses/by-nc/4.0) which permits unrestricted noncommercial use, distribution, and reproduction in any medium, provided the original work is properly cited.

Copyright $\odot 2015$ by Korean Academy of Rehabilitation Medicine ease, with a high prevalence that increases with age $[1,2]$. The most commonly affected large weight-bearing joints are the knees, where the disease is particularly disabling, since it causes difficulty in rising from a chair, climbing stairs, standing and walking [3].

Pain is the main reason for OA patients to seek clinical services [4]. The management of early-stage OA is fundamental for effective symptom relief, management of the resulting functional limitations, and enhancing the health-related quality of life.

The treatment methods for knee OA are diverse. Biomechanical interventions, intra-articular corticosteroids, exercise (land-based and water-based), self-management and education, strength training, weight management, 
acetaminophen, balneotherapy, capsaicin, mobility aids, duloxetine, oral non-steroidal anti-inflammatory drugs (NSAIDs) and topical NSAIDs are recommended in the 2010 Osteoarthritis Research Society International (OARSI) guidelines [5]. However, the results of these treatments are not consistent, and patients with OA continue to experience pain [4]. Therefore, we are interested formulating in new strategies to reduce pain and improve function.

Extracorporeal shock wave therapy (ESWT) was first used to break kidney stones. Its use was proposed for musculoskeletal disorders as the result of an incidental observation of an osteoblastic response pattern, during animal studies, in the late 1980s [6]. In recent years, ESWT has been widely used for pain relief and the treatment of musculoskeletal disorders, such as epicondylitis, plantar fasciitis, calcific tendinitis, and so on [7-9]. The procedure is non-invasive, has a low instance of complication and does not require hospitalization. Veterinarians first began to use ESWT to treat equine knee OA [10]. Several studies found that this procedure showed reduced progression of $\mathrm{OA}$, with decreased articular cartilage degradation and improved motor dysfunction, as well as pain relief in animals with OA [11-13]. In recent years, Zhao et al. [14] have investigated the efficacy of ESWT in human knee OA. They determined that ESWT is effective in reducing pain and improving knee function; however, they did not establish a treatment protocol for ESWT, regarding the proper amount of energy.

The purpose of this study was, therefore to investigate the dose-related effects of ESWT (at different total energy flux density [EFD]) in patients with knee OA.

\section{MATERIALS AND METHODS}

\section{Subjects}

All patients who had unilateral knee pain, from January 2013 to December 2013, were recruited. The inclusion criteria of the subjects were 1 ) aged $>45 ; 2$ ) diagnosed as symptomatic knee OA for at least 3 months, according to the clinical criteria of the American College of Rheumatology [15];3) their radiographic findings had to be compatible with knee OA, with a Kellgren-Lawrence (K-L) grade II or III in a simple X-ray [16]; and 4) had tenderness on medial tibial plateau area.

We excluded patients who had comorbidities, such as any neurologic disorders or systemic disease, previous surgical intervention or intra-articular injection in the last 6 months, any contraindication to magnetic resonance imaging or radiography, or trauma history on knee.

Seventy-five subjects were recruited, 60 of which met the inclusion criteria. Written informed consent was obtained from all subjects prior to the study. The study was performed in accordance with the principles set forth in the Declaration of Helsinki.

\section{Methods}

\section{Extracorporeal shock wave therapy}

We used block randomization via a computerized program. An independent researcher assigned the study subjects to either the low-energy group (group L: $\mathrm{n}=30$, 1,000 shocks/session, EFD per shock $0.040 \mathrm{~mJ} / \mathrm{mm}^{2}$ ) or the medium-energy group (group $\mathrm{M}: \mathrm{n}=30,1,000$ shocks/ session, EFD per shock $0.093 \mathrm{~mJ} / \mathrm{mm}^{2}$ ), but was not involved in the treatment or the outcome assessment. All subjects received three sessions of ESWT, at once-weekly intervals, at different energy level according to their groups (Fig. 1).

We used the Dornier Epos Ultra (Dornier MedTech, Kennesaw, GA, USA), which was approved in 2001. Our ESWT has an electromagnetic source, and the type of ESWT is the smart focus type. The ESWT was executed by physician who was not involved in the selection and assessment of the patients. At each treatment session, all subjects were positioned in a supine manner, with the affected knee flexed at $90^{\circ}$. The shockwave probe was held stationary on a tender area around the medial tibial plateau. To reduce the loss of shockwave energy at the interface, an aqueous gel was applied between the probe and the skin. The practitioner frequently checked the treatment areas of the patients during the therapy and excluded those who developed side effects on the treatment area, such as bruising or edema. During the course of the experiment, all subjects were prevented from receiving any additional treatment, such as physical therapy, steroid injection, or anti-inflammatory drugs. No bed rest was required after treatment, but a low level of physical activity was recommended for the following 48 hours.

\section{Outcome measures}

The clinical assessments included an assessment of 


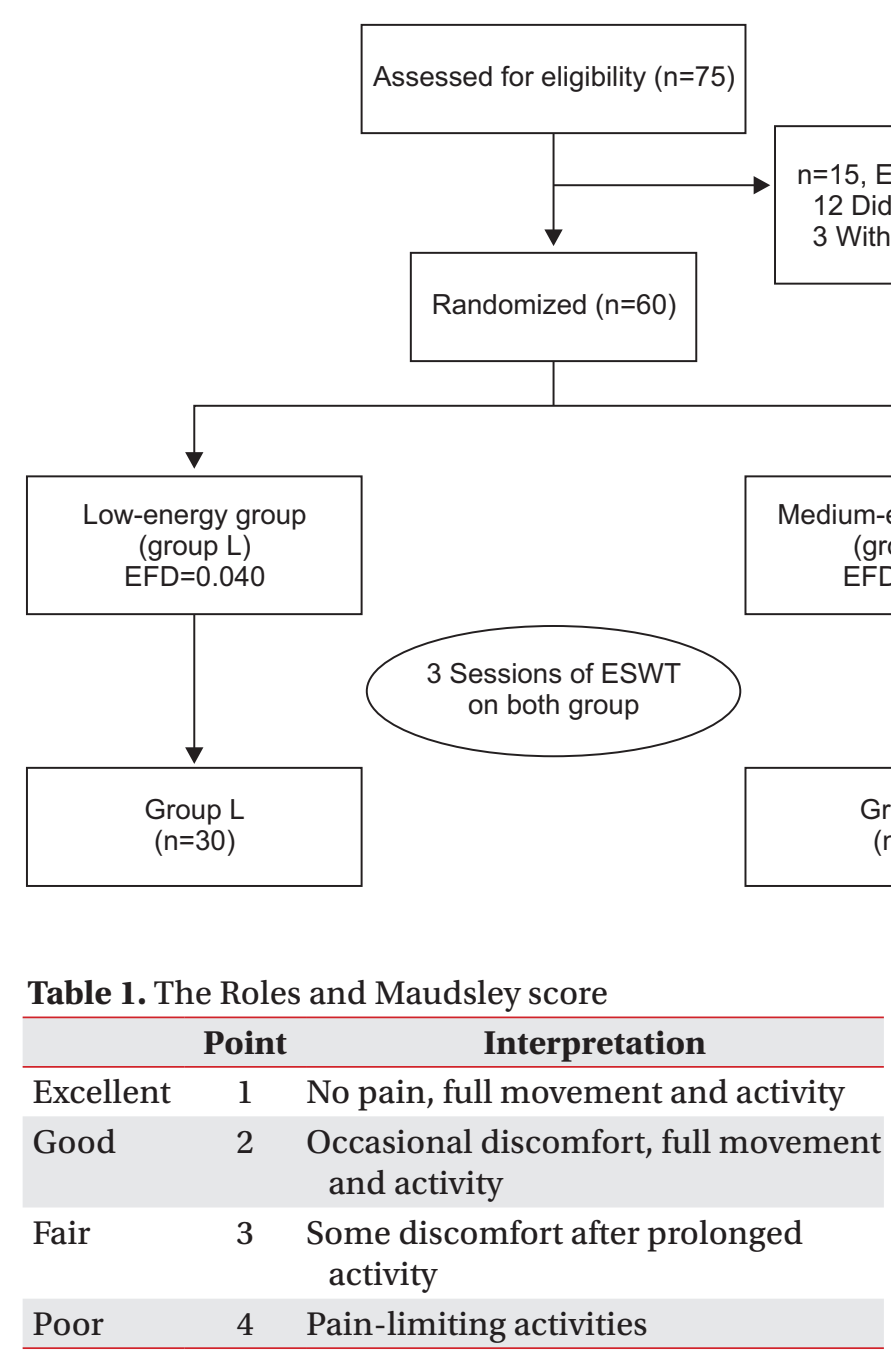

pain, on movement, and a physical function assessment. The primary outcome measure was 'pain on movement', which was measured using a visual analogue scale (VAS) [17], with 0 indicating no pain and 10 indicating maximum pain. The VAS scores were measured in relation to the degree of pain that patients experienced during their usual daily activity. The secondary outcome measure was 'disability' on the Roles and Maudsley (RM) score, the Western Ontario and McMaster University Osteoarthritis Index (WOMAC), and the Lequesne index. All assessments were performed at the baseline and at 1,4 , and 12 weeks after ESWT by a physician who was not involved in the selection and treatment of patients.

The RM score was used to assess functional evaluation [18]. It was recorded using four categories, based on the pain and activity ranges in daily life: excellent, good, fair, and poor (Table 1).
The WOMAC assesses the symptoms of OA and is a validated disease-specific self-reporting questionnaire that refers to the 48 hours before assessment [19]. The index consists of five questions covering the severity of the knee pain two questions covering stiffness and 17 questions covering limitations in physical function. The WOMAC score ranges from 0 (best) to 96 (worst), with high score representing worse symptom severity.

The disability of patients with knee OA was assessed using the Lequesne index. The questionnaire included 11 questions regarding knee discomfort, endurance of ambulation, and difficulties in daily life [20]. A maximum score of 26 indicated the greatest degree of dysfunction, and a score of 1-3 indicated a mild dysfunction. A score of less than 7 points indicated an acceptable functional status.

\section{Statistical analysis}

A Student t-test was used to compare the homogeneity of the baseline characteristics between two groups. The treatment effects (over time) within each group and the differences in the treatment effects between the two groups were examined using repeated measure ANOVA. All analyses were performed with the SPSS ver. 18.0 (SPSS Inc., Chicago, IL, USA). A p-value of $<0.05$ was considered statistically significant. 
Table 2. The demographic and baseline clinical characteristics of patients

\begin{tabular}{lccc}
\hline & Group L $(\mathbf{n}=\mathbf{3 0})$ & Group M $^{(\mathbf{n}=\mathbf{3 0})}$ & p-value $^{\text {a) }}$ \\
\hline Sex (male:female) & $26: 4$ & $27: 3$ & \\
Age $(\mathrm{yr})$ & $65.1 \pm 6.3$ & $63.5 \pm 5.4$ & 0.293 \\
Height $(\mathrm{cm})$ & $167.2 \pm 9.5$ & $168.3 \pm 7.9$ & 0.742 \\
Weight $(\mathrm{kg})$ & $68.5 \pm 5.3$ & $70.3 \pm 6.8$ & 0.631 \\
BMI $\left(\mathrm{kg} / \mathrm{m}^{2}\right)$ & $23.8 \pm 1.7$ & $24.2 \pm 2.1$ & 0.783 \\
Duration $(\mathrm{mo})$ & $19.3 \pm 5.2$ & $16.7 \pm 4.7$ & 0.685 \\
\hline VAS & $5.93 \pm 1.15$ & $6.10 \pm 1.26$ & 0.643 \\
Roles and Maudsley score & $3.27 \pm 0.76$ & $3.23 \pm 0.73$ & 0.865 \\
\hline WOMAC & $56.90 \pm 11.44$ & $57.63 \pm 10.21$ & 0.794 \\
\hline Lequesne index & $14.5 \pm 3.5$ & $14.9 \pm 3.1$ & 0.856 \\
\hline
\end{tabular}

Values are presented as number or mean \pm standard deviation.

Group L, low-energy $\left(0.040 \mathrm{~mJ} / \mathrm{mm}^{2}\right)$ group; group $\mathrm{M}$, medium-energy $\left(0.093 \mathrm{~mJ} / \mathrm{mm}^{2}\right)$ group; BMI, body mass index; VAS, visual analogue scale; WOMAC, Western Ontario and McMaster University Osteoarthritis Index.

${ }^{\text {a) }}$ Student t-test.

\section{RESULTS}

\section{Patient characteristics}

Within the group of 60 patients, 53 were male and 7 were female. Their average age was $64.3 \pm 5.8$ years old, their average height was $167.8 \pm 8.5 \mathrm{~cm}$, and their average weight was $69.4 \pm 6.1 \mathrm{~kg}$. The average body mass index (BMI) was $24.0 \pm 1.8 \mathrm{~kg} / \mathrm{m}^{2}$ and the average disease duration was $18.0 \pm 4.9$ months. The average VAS, RM, and WOMAC scores were $6.02 \pm 1.15$ points, $3.25 \pm 0.75$ points, and $57.27 \pm 10.75$ points, respectively. The average Lequesne index was $14.7 \pm 3.3$.

There were no significant differences in sex, age, height, weight, BMI, disease duration, VAS score, RM score, WOMAC score, or Lequesne index between the two groups prior to the treatments (Table 2). There were no cases in which the treatment was discontinued due to the occurrence of side effects or complications, although some patients complained of minor discomforts like transient skin reddening or swelling.

\section{Changes in pain after the ESWT}

At the baseline, the VAS scores in group $\mathrm{M}$ and group L were $6.10 \pm 1.26$ and $5.93 \pm 1.15$, respectively. After 1 week, the VAS scores in each group were $5.13 \pm 0.82$ and $4.67 \pm 0.48$. After 4 weeks, were $2.73 \pm 0.83$ and $2.77 \pm 0.57$, and after 12 weeks, they were $1.57 \pm 0.50$ and $1.90 \pm 0.71$, respectively. Compared to the baseline, the VAS score significantly decreased with time, up to the 12 -week follow-

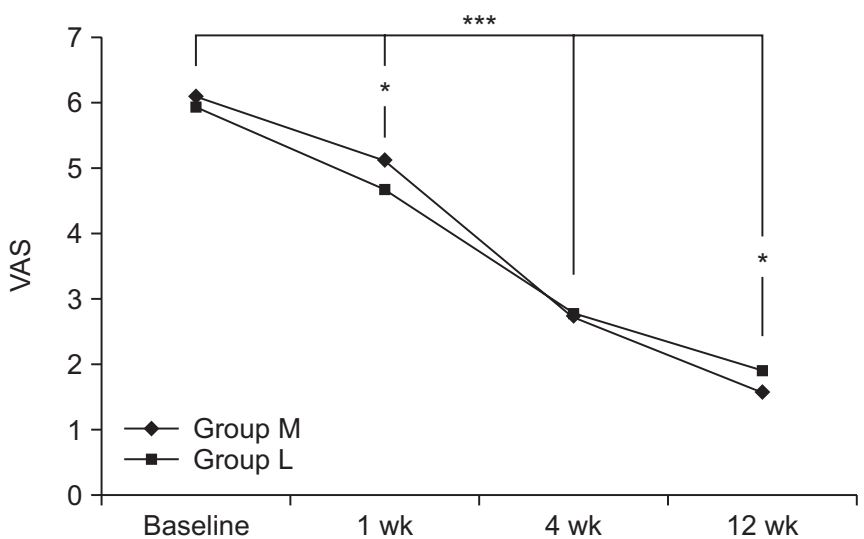

Fig. 2. The visual analogue scale (VAS) score at the baseline and 1-, 4-, and 12-week follow-ups after the extracorporeal shock wave therapy in both groups. This figure shows significant improvement for both groups, in the VAS score, for entire period $\left({ }^{* * *} \mathrm{p}<0.001\right.$ for time effect, ${ }^{* * *} \mathrm{p}<0.001$ for group-time interaction) and significant differences between the two groups at 1 week and 12 weeks $\left({ }^{*} \mathrm{p}<0.05\right)$. Group $\mathrm{M}$, medium-energy $\left(0.093 \mathrm{~mJ} / \mathrm{mm}^{2}\right)$ group; group $\mathrm{L}$, low-energy $\left(0.040 \mathrm{~mJ} / \mathrm{mm}^{2}\right)$ group.

up for both groups $(\mathrm{p}<0.001$ for time effect, $\mathrm{p}<0.001$ for group-time interaction). There were also significant differences between the two groups at 1 week and 12 weeks $(\mathrm{p}<0.05)$ (Fig. 2).

\section{Changes in function after the ESWT}

At the baseline, the RM scores in the two groups were $3.23 \pm 0.73$ and $3.27 \pm 0.76$, respectively. After 1 week, 


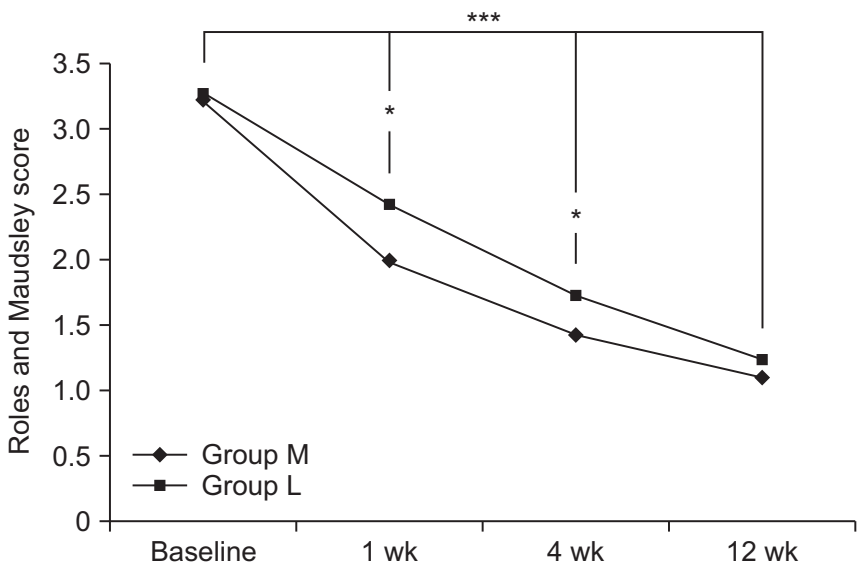

Fig. 3. The Roles and Maudsley score at the baseline and 1-, 4-, 12-week follow-ups after the extracorporeal shock wave therapy, in both groups. This figure shows significant improvement for both group in the Roles and Maudsley score over the entire period $\left({ }^{* * *} \mathrm{p}<0.001\right.$ for time effect, ${ }^{* * *}$ p $<0.05$ for group-time interaction) and significant differences between the two groups at 1 week and 4 weeks $\left({ }^{*} \mathrm{p}<0.05\right)$. Group M, medium-energy $(0.093 \mathrm{~mJ} /$ $\mathrm{mm}^{2}$ ) group; group L, low-energy $\left(0.040 \mathrm{~mJ} / \mathrm{mm}^{2}\right)$ group.

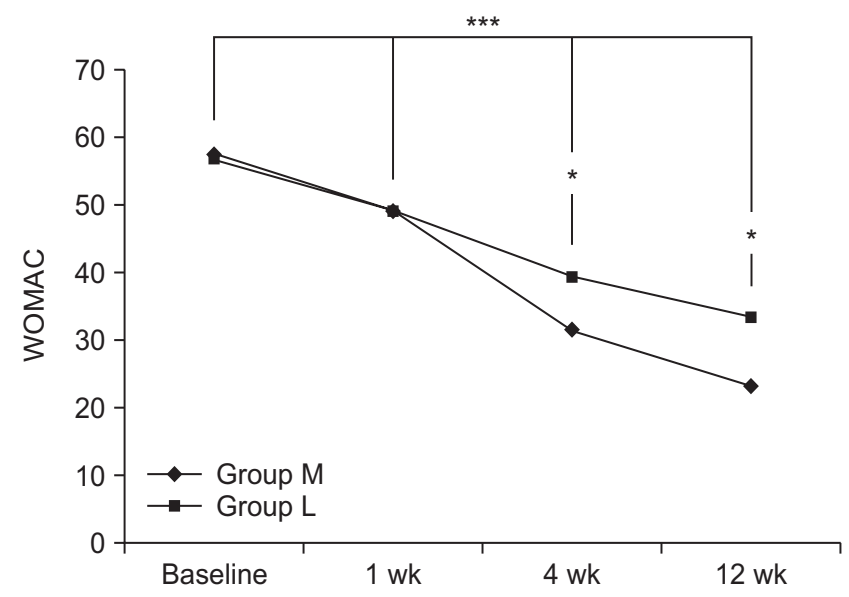

Fig. 4. The Western Ontario and McMaster University Osteoarthritis Index (WOMAC) score at the baseline and 1-, 4-, 12-week follow-ups after the extracorporeal shock wave therapy, in both groups. This figure shows significant improvement, for both groups, in WOMAC score over the entire period $\left({ }^{* * *} \mathrm{p}<0.001\right.$ for time effect, ${ }^{* * *} \mathrm{p}<0.001$ for group-time interaction), and significant differences between the two groups at 4 weeks and 12 weeks $\left({ }^{*} \mathrm{p}<0.05\right)$. Group M, medium-energy $(0.093 \mathrm{~mJ} /$ $\left.\mathrm{mm}^{2}\right)$ group; group L: low-energy $\left(0.040 \mathrm{~mJ} / \mathrm{mm}^{2}\right)$ group.

the RM scores in the two groups were $2.00 \pm 0.53$ and $2.43 \pm 0.57$, respectively. After 4 weeks, they were $1.43 \pm 0.50$

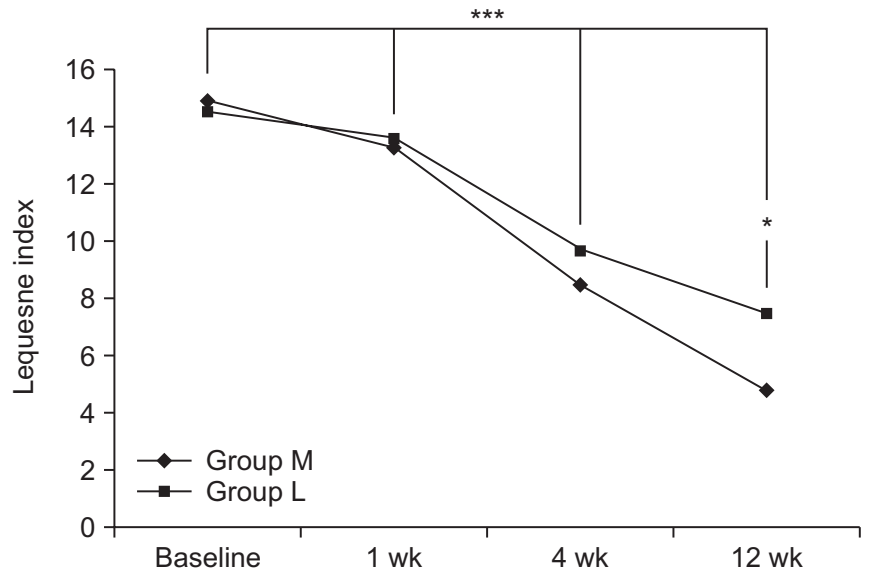

Fig. 5. The Lequesne index at the baseline and 1-, 4-, 12week follow-ups after extracorporeal shock wave therapy, for both groups. This figure shows significant improvement, for both groups, in the Lequesne index over entire period $\left({ }^{* * *} \mathrm{p}<0.001\right.$ for time effect, ${ }^{* * *} \mathrm{p}<0.001$ for grouptime interaction), and significant differences between the two groups at 12 weeks $\left({ }^{*} \mathrm{p}<0.05\right)$. Group $\mathrm{M}$, mediumenergy $\left(0.093 \mathrm{~mJ} / \mathrm{mm}^{2}\right)$ group; group L: low-energy (0.040 $\mathrm{mJ} / \mathrm{mm}^{2}$ ) group.

and $1.73 \pm 0.64$, and after 12 weeks, they were $1.10 \pm 0.31$ and $1.23 \pm 0.43$, respectively. Compared to the baseline, the RM score significantly decreased with time, up to the 12-week follow-up, in both groups $(\mathrm{p}<0.001$ for time effect, $\mathrm{p}<0.05$ for group-time interaction); there were significant differences between the two groups at 1 week and 4 weeks $(\mathrm{p}<0.05)$ (Fig. 3).

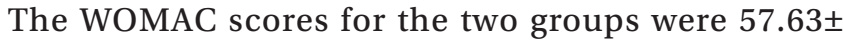
10.21 and $56.90 \pm 11.44$ at baseline, respectively. After 1 week, WOMAC scores for the two groups were $49.10 \pm 12.23$ and $49.17 \pm 9.77$, respectively. After 4 weeks, they were $31.57 \pm 9.07$ and $39.27 \pm 7.15$, after 12 weeks, they were $23.20 \pm 7.78$ and $33.33 \pm 7.03$, respectively. Compared to the baseline, the WOMAC score significantly decreased with time, up to the 12-week follow-up, in both groups ( $\mathrm{p}<0.001$ for time effect, $\mathrm{p}<0.001$ for group-time interaction). There were significant differences between the two groups at 4 weeks and 12 weeks $(\mathrm{p}<0.05)$ (Fig. 4).

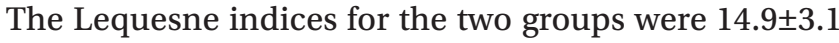
and $14.5 \pm 3.5$, respectively, at the baseline. After 1 week, they were $13.3 \pm 2.8$ and $13.6 \pm 2.9$, respectively; after 4 weeks, they were $8.5 \pm 3.3$ and $9.7 \pm 2.5$, and after 12 weeks they were $4.8 \pm 1.5$ and $7.5 \pm 2.2$, respectively. Compared to the baseline, the Lequesne index significantly decreased, with time, up to the 12 week follow-up in both groups 
( $p<0.001$ for time effect, $\mathrm{p}<0.001$ for group-time interaction). There were significant differences between the two groups at 12 weeks $(p<0.05)$ (Fig. 5).

\section{DISCUSSION}

The nonsurgical management of knee $\mathrm{OA}$ is essential for effective symptom relief and the management of functional limitations [21]. In January 2010, the OARSI published an update to their recommendations for the treatment of knee OA [5]. In recent years, ESWT has been the leading therapeutic choice for chronic tendinopathy, nonunion of a long bone fracture, and the early stage of avascular necrosis of the femoral head [6]. More recently, the use of ESWT has expanded to include the treatment of OA in animals, and has shown improved motor function and pain management [11-13]. Some studies have shown the efficacy of ESWT for knee OA patients, by reducing pain and improving knee function [14].

We considered that ESWT could have a beneficial effect for the pain and physical function in patients with knee OA. However, the exact mechanisms of ESWT, on knee $\mathrm{OA}$, are complex and have not been clearly explained. The mechanism of ESWT causes selective dysfunction of sensory unmyelinated nerve fibers [22]. It is known to improve the symptoms of OA via an inflammatory response triggered by the secretion of growth factors, and also to repair damaged tissues by encouraging angiogenesis [2325]. The levels of neuropeptide calcitonin gene-related peptides were decreased in the dorsal root ganglion in a rat OA knee model, after the ESWT treatment; this peptide is expressed by nociceptors and is thought to play a role in the sensation of joint pain [12]. ESWT could reduce the progression of OA in animal models $[13,26,27]$. The application of ESWT, for a damaged anterior cruciate ligament in rats, improved subchondral bone remodeling and decreased cartilage degradation [26]. Moreover, the application of ESWT reduced the progression of OA in rabbits, which may be related to the decreased levels of nitric oxide, and is likely mediated by a reduced chondrocyte apoptosis [13].

The ESWT can be divided into different energy influx levels. Some previous studies have divided the ESWT treatment intensity into three levels, which are 1) low intensity (EFD, $<0.08 \mathrm{~mJ} / \mathrm{mm}^{2}$ ); 2) medium intensity (EFD, $0.08-0.28 \mathrm{~mJ} / \mathrm{mm}^{2}$ ); and 3) high intensity (EFD, >0.28 mJ/ $\left.\mathrm{mm}^{2}\right)[28,29]$.

While some studies that advocate the use of high intensity energy report that it requires only a single treatment, and that the treatment effects are superior [30], the increased energy influx results in a corresponding increase in pain, local swelling, and tenderness. Therefore, higher intensity treatments usually require local anesthesia, which is known to reduce the efficacy of the treatment [31]. Some basic scientific studies have shown that the ESWT application, for OA, is safe with proper dosing [32]. EFD, applied at $>0.50 \mathrm{~mJ} / \mathrm{mm}^{2}$, caused degenerative changes in the hyaline cartilage of rats [33]. In addition, by using lower energy, it is possible to prevent patients from having to adapt (quite as much) to the treatment, and also to prevent patients from giving up on their treatment outright as a result of the pain caused by treatments using high energy.

In our study, the treatment effects in the two groups were compared: group L had low energy $\left(0.040 \mathrm{~mJ} / \mathrm{mm}^{2}\right)$ and group $\mathrm{M}$ had medium energy $\left(0.093 \mathrm{~mJ} / \mathrm{mm}^{2}\right)$, which was applied over three sessions. Our results show that each group improved significantly by the 12-week followup, in terms of the pain and functional scores, compared to the baseline scores. The medium energy group showed a better pain score than the low energy group at 1 week and 12 weeks, a better RM score at 1 week and 4 weeks, a better WOMAC score at 4 weeks and 12 weeks, and a better Lequesne index at 12 weeks. This concurs with the results of previous studies, which demonstrated that higher intensity energy destroys more unmyelinated sensory nerve fibers, and thereby has a greater pain-reducing effect $[22,34]$. The histological reaction to the ESWT is known to be dose-dependent on the total energy, so medium energy group shows greater improvement $[35,36]$.

This study has several limitations. For example, the control group was not employed to exclude the placebo effect. The number of patients was relatively small, and the evaluation period (at 12 weeks) was too short to assess fully the long-term effectiveness. An explanation on the principles and mechanisms of the effectiveness of the ESWT are provided in the inferences from past studies. Further studies will be needed to confirm these findings.

In conclusion, the adaptation of the medium energy ESWT was more efficient, in terms of relieving pain and restoring functional outcome, than the low energy ESWT. Therefore, EFD can be considered a significant influenc- 
ing factor, when treating knee OA with ESWT.

\section{CONFLICT OF INTEREST}

No potential conflict of interest relevant to this article was reported.

\section{REFERENCES}

1. Dunlop DD, Manheim LM, Song J, Chang RW. Arthritis prevalence and activity limitations in older adults. Arthritis Rheum 2001;44:212-21.

2. Dawson J, Linsell L, Zondervan K, Rose P, Randall T, Carr A, et al. Epidemiology of hip and knee and its impact on overall health status in older adults. Rheumatology (Oxford) 2004;43:497-504.

3. Peat G, McCamey R, Croft P. Knee pain and osteoarthritis in older adults: a review of community burden and current use of primary healthcare. Ann Rheum Dis 2001;60:91-7.

4. Sofat N, Ejindu V, Kiely P. What makes osteoarthritis painful? The evidence for local and central pain processing. Rheumatology (Oxford) 2011;50:2157-65.

5. McAlindon TE, Bannuru RR, Sullivan MC, Arden NK, Berenbaum F, Bierma-Zeinstra SM, et al. OARSI guidelines for the non-surgical management of knee osteoarthritis. Osteoarthr Cartil 2014;22:363-88.

6. Wang CJ. Extracorporeal shockwave therapy in musculoskeletal disorders. J Orthop Surg Res 2012;7:11.

7. Wang CJ, Yang KD, Wang FS, Chen HH, Wang JW. Shock wave therapy for calcific tendinitis of the shoulder: a prospective clinical study with two-year followup. AM J Sports Med 2003;31:425-30.

8. Rompe JD, Theis C, Maffulli N. Shock wave treatment for tennis elbow. Orthopade 2005;34:567-70.

9. Rompe JD. Shock-wave therapy for plantar fasciitis. J Bone Joint Surg Am 2005;87:681-2.

10. Revenaugh MS. Extracorporeal shock wave therapy for treatment of osteoarthritis in the horse: clinical applications. Vet Clin North Am Equine Pract 2005;21:60925.

11. Frisbie DD, Kawcak CE, McIlwraith CW. Evaluation of the effect of extracorporeal shock wave treatment on experimentally induced osteoarthritis in middle carpal joints of horses. AM J Vet Res 2009;70:449-54.

12. Ochiai N, Ohtori S, Sasho T, Nakagawa K, Takahashi K,
Takahashi N, et al. Extracorporeal shock wave therapy improves motor dysfunction and pain originating from knee osteoarthritis in rats. Osteoarthr Cartil 2007;15:1093-6.

13. Zhao Z, Ji H, Jing R, Liu C, Wang M, Zhai L, et al. Extracorporeal shock-wave therapy reduces progression of knee osteoarthritis in rabbits by reducing nitric oxide level and chondrocyte apoptosis. Arch Orthop Trauma Surg 2012;132:1547-53.

14. Zhao Z, Jing R, Shi Z, Zhao B, Ai Q, Xing G. Efficacy of extracorporeal shockwave therapy for knee osteoarthritis: a randomized controlled trial. J Sur Res 2013;185:661-6.

15. Altman R, Asch E, Bloch D, Bole G, Borenstein D, Brandt $\mathrm{K}$, et al. Development of criteria for the classification and reporting of osteoarthritis: classification of osteoarthritis of the knee. Arthritis Rheum 1986;29:1039-49.

16. Kellgren JH, Lawrence JS. Radiological assessment of osteo-arthrosis. Ann Rheum Dis 1957;16:494-502.

17. Carlsson AM. Assessment of chronic pain. I. Aspects of the reliability and validity of the visual analogue scale. Pain 1983;16:87-101.

18. Roles NC, Maudsley RH. Radial tunnel syndrome: resistant tennis elbow as a nerve entrapment. J Bone Joint Surg Br 1972;54:499-508.

19. Bellamy N, Campbell J, Stevens J, Pilch L, Stewart C, Mahmood Z. Validation study of a computerized version of the Western Ontario and McMaster Universities VA3.0 Osteoarthritis Index. J Rheumatol 1997;24:2413-5.

20. Lequesne M. Clinical feature, diagnostic criteria, functional assessments and radiological classifications of osteoarthritis. Rheumatology 1982;7:1-10.

21. Kon E, Filardo G, Drobnic M, Madry H, Jelic M, Dijk $\mathrm{N}$, et al. Non-surgical management of early knee osteoarthritis. Knee Surg Sports Traumatol Arthrosc 2012;20:436-49.

22. Takahashi N. Ohtori S, Saisu T, Moriya H, Wada Y. Second application of low-energy shock waves has a cumulative effect on free nerve endings. Clin Orthop Relat Res 2006;443:315-9.

23. Speed CA. Extracorporeal shock-wave therapy in the management of chronic soft-tissue conditions. J Bone Joint Surg 2004;86:165-71.

24. Thiel M. Application of shock waves in medicine. Clin 
Orthop Relat Res 2001;387:18-21.

25. Wang CJ, Wang FS, Yang KD, Weng LH, Hsu CC, Huang CS, et al. Shock wave therapy induces neovascularization at the tendon-bone junction: a study in rabbits. J Orthop Res 2003;21:984-9.

26. Wang CJ, Weng LH, Ko JY, Wang JW, Chen JM, Sun YC, et al. Extracorporeal shockwave shows regression of osteoarthritis of the knee in rats. J Surg Res 2011;171:601-8.

27. Wang CJ, Sun YC, Wong T, Hsu SL, Chou WY, Chang HW. Extracorporeal shockwave therapy shows timedependent chondroprotective effects in osteoarthritis of the knee in rats. J Surg Res 2012;178:196-205.

28. Rompe JD, Furia J, Weil L, Maffulli N. Shock wave therapy for chronic plantar fasciopathy. Br Med Bull 2007;81-82:183-208.

29. Gollwitzer H, Diehl P, von Korff A, Rahlfs VW, Gerdesmeyer L. Extracorporeal shock wave therapy for chronic painful heel syndrome: a prospective, double blind, randomized trial assessing the efficacy of a new electromagnetic shock wave device. J Foot Ankle Surg 2007;46:348-57.

30. Metzner G, Dohnalek C, Aigner E. High-energy extracorporeal shock-wave therapy for the treatment of chronic plantar fasciitis. Foot Ankle Int 2010;31:790-6.
31. Rompe JD, Meurer A, Nafe B, Hofmann A, Gerdesmeyer L. Repetitive low-energy shock wave application without local anesthesia is more efficient than repetitive low-energy shock wave application with local anesthesia in the treatment of chronic plantar fasciiitis. J Orthop Res 2005;23:931-41.

32. Renz H, Rupp S. Effects of shock waves on chondrocytes and their relevance in clinical practice. Arch Orthop Trauma Surg 2009;129:641-7.

33. Mayer-Wagner S, Ernst J, Maier M, Chiquet M, Joos H, Muller PE, et al. The effect of high-energy extracorporeal shock waves on hyaline cartilage of adult rats in vivo. J Orthop Res 2010;28:1050-6.

34. Ohtori S, Inoue G, Mannoji C, Saisu T, Takahashi K, Mitsuhashi S, et al. Shock wave application to rat skin induces degeneration and reinnervation of sensory nerve fibers. Neurosci Lett 2001;315:57-60.

35. McClure S, Dorfmuller C. Extracorporeal shock wave therapy: theory and equipment. Clin Tech Equine Pract 2003;2:348-57.

36. Rompe JD, Kirkpatrick CJ, Kullmer K, Schwitalle M, Krischek O. Dose-related effects of shock waves on rabbit tendo achillis: a sonographic and histological study. J Bone Joint Surg Br 1998;80:546-52. 